\title{
Tunable Transition Metal-Ligand Complexation for Enhanced Elucidation of Flavonoid Diglycosides by Electrospray Ionization Mass Spectrometry
}

\author{
Michael Pikulski, Apolonio Aguilar, and Jennifer S. Brodbelt \\ Department of Chemistry and Biochemistry, The University of Texas at Austin, Austin, Texas, USA
}

A tunable ESI-MS/MS strategy for differentiation of flavone and flavanone diglycoside isomers based on metal complexation with auxiliary ligands is reported. The addition of a metal salt and an auxiliary ligand to a flavonoid solution results in the formation of [M(II) (flavonoid-H) auxiliary ligand $]^{+}$complexes, where $\mathrm{M}(\mathrm{II})$ is a transition metal. A series of auxiliary ligands with electron-withdrawing substituents were synthesized to tailor the relative metal binding affinities of the ligands and thus directly influence the stabilities, and consequently the dissociation pathways, of the complexes. Upon collisionally activated dissociation, the complexes yield fragmentation patterns in which the abundances of key diagnostic ions are enhanced, thus facilitating isomer differentiation. (J Am Soc Mass Spectrom 2007, 18, 422-431) (c 2007 American Society for Mass Spectrometry

$\mathrm{D}$ ifferentiation of isomers remains a challenging analytical problem and confirming structures of isomers is difficult even when they can be adequately separated from mixtures. Tandem mass spectrometry has been used extensively for structural elucidation of isomers [1-12] and there continues to be interest in applying existing tandem mass spectrometric methods, such as $\mathrm{MS}^{\mathrm{n}}[9,13,14]$, and developing new ones, such as STEP $[15,16]$, a statistically based method that relies on comparison of abundances of fragment ions obtained for different collision activation conditions, to differentiate isomers. Our group has focused on the development of metal complexation strategies to enhance the differentiation of isomers [17-23] because metal complexation often alters the dissociation pathways of ions, thus giving additional diagnostic fragment ions. We have reported several metal complexation strategies that have been useful for distinguishing flavonoid isomers and, in this study, we expand this approach by developing a "tunable" collisionally activated dissociation (CAD) method based on the use of rationally selected auxiliary ligands to form metal complexes. The concept of this tunable CAD strategy is applied for the differentiation of flavonoid isomers.

Flavonoids are phytochemicals found in almost all plants, including fruits and vegetables. Several studies have indicated their antioxidant, chemopreventive, antiviral, antibacterial, and radical-scavenging properties

Published online November 16, 2006

Address reprint requests to Dr. Jennifer S. Brodbelt, Department of Chemistry and Biochemistry, The University of Texas at Austin, 1 University Station A5300, Austin, TX 78712-0165. E-mail: jbrodbelt@mail.utexas.edu
[24-28]; moreover, they have been implicated in cardiovascular protection [29-36]. Although all flavonoids are structurally similar, many exist as glycosides and there are subtle differences in their structure that lead to important changes in their biological activity. These differences include hydroxylation position of the aglycon, glycosylation site, sequence of glycosylation, and interglycosidic linkages of the glycan portion. It is because of these subtle differences that there is a need for sensitive analytical techniques to elucidate these similar compounds.

Mass spectrometry has become an ideal tool for the analysis of flavonoids, and two recent reviews provide an excellent background of the techniques used and the analyses performed [37, 38]. Electrospray ionization tandem mass spectrometry (ESI-MS/MS) has been used extensively to differentiate several sets of flavonoid isomers over the past decade. For example, for a series of flavonoid diglycosides, CAD has been used to pinpoint the intersaccharide linkages (rutinosides: $1 \rightarrow 6$ disaccharides versus neohesperidosides: $1 \rightarrow 2$ disaccharides) in flavonoid-O-glycosides based on relative abundances of the fragment ions obtained from protonated flavonoids [7]. However, in general it has been noted that flavonoids do not protonate particularly well because they are acidic, phenolic compounds. Although flavonoids deprotonate efficiently, the resulting fragmentation patterns often do not give diagnostic ions adequate for differentiation of isomers. Metal complexation is an alternative ionization strategy that has been explored in our group for improved analysis of flavonoids [17-23], and we have explored metal complexation both with and without the use of an auxiliary 
ligand to produce stable complexes. For example, using an auxiliary ligand, typically a pyridyl-type chelating agent, complexes of the type [M(II) (flavonoid - H) auxiliary ligand $]^{+}$are formed. Metal complexation not only provides greater ion abundances but also enhances the structural differentiation of isomeric flavonoids.

In one of our previous studies involving flavonoid diglycosides, the impact of the auxiliary ligand on the abundances of metal complexes and the resulting fragmentation pathways of the complexes was evaluated [21]. It was demonstrated that the nature of the auxiliary ligand affected the types of fragment ions and their abundances in the CAD mass spectra. Specifically, it was found that as the alkyl substituents of the ligands changed (that is, 1,10-phenanthroline versus 4,7-diphenyl1,10-phenanthroline), the abundances of several diagnostic fragment ions observed for flavone rutinoside and neohesperidoside isomeric pairs varied systematically. This result revealed that it was possible to tune the CAD spectra by careful selection of the auxiliary ligand. However, the only suitable commercially available auxiliary ligands were those with electron-releasing alkyl substituents, thus limiting the scope of the study. Electron-releasing substituents increase the metal binding energies of pyridyl ligands and therefore in some cases destabilize the desired [M(II) (flavonoid - $\mathrm{H}$ ) pyridyl ligand] ${ }^{+}$complexes. Pyridyl ligands have intrinsically high metal binding energies, both in solution [39-43] and in the gas-phase [44, 45], arising from their ability to chelate metal ions by their nitrogen atoms. Addition of electron-withdrawing substituents should temper the metal binding energies and thus expand the options for creating tunable auxiliary ligands for the differentiation of isomers, such as flavonoids, by metal complexation strategies.

In this study, we explore the effect of incorporating electron-withdrawing substituents into the pyridyl ligands for metal complexation, evaluated in terms of the success of differentiating flavonoid isomers. The halogens are interesting substituents because of their inductive electron-withdrawing effects. For assessment of the method, 12 isomers of 7-O-flavonoid diglycosides are evaluated, including three isomeric pairs from the flavone class (both $1 \rightarrow 2$ versus $1 \rightarrow 6$ disaccharide linkages) and three pairs from the flavanone class $(1 \rightarrow 2$ versus $1 \rightarrow 6$ ) (Figure 1 ).

\section{Experimental}

\section{Chemical Reagents}

The flavonoids rhoifolin, isorhoifolin, neodiosmin, diosmin, and narirutin were purchased from Indofine (Somerville, NJ, USA). Fortunellin, linarin, poncirin, and didymin were purchased from Extrasynthese (Genay, France). Naringin, neohesperidin, hesperidin, $\mathrm{CoBr}_{2}$, $\mathrm{CuBr}_{2}$, 2,2'-bipyridine, 6,6'-dibromo-2,2'-bipyridine, 4,4'dimethyl-2,2'-bipyridine, 1,10-phenanthroline, and 5- nitro-1,10-phenanthroline were purchased from Sigma (St. Louis, MO, USA).

\section{Ligand Syntheses}

4,4'-Dibromo-2,2'-bipyridine was synthesized from 2,2'bipyridine according to a literature procedure $[46,47]$ and was purified using silica gel flash chromatography. ${ }^{1} \mathrm{H} \mathrm{NMR}\left(\mathrm{CDCl}_{3}\right) \delta=7.509(2 \mathrm{H}, \mathrm{dd}, \mathrm{J}=5.2 \mathrm{~Hz}, 1.6 \mathrm{~Hz})$, $8.486(2 \mathrm{H}, \mathrm{d}, \mathrm{J}=5.2 \mathrm{~Hz}), 8.608(2 \mathrm{H}, \mathrm{d}, \mathrm{J}=1.6 \mathrm{~Hz})$.

$4,4^{\prime}$-Bis(bromomethyl)-2,2'-bipyridine was synthesized from $4,4^{\prime}$-dimethyl-2,2' -bipyridine according to a literature procedure [48] and was purified using silica gel flash chromatography. ${ }^{1} \mathrm{H}$ NMR $\left(\mathrm{CD}_{3} \mathrm{Cl}_{3}\right) \delta=7.507$ $(2 \mathrm{H}, \mathrm{d}, \mathrm{J}=8 \mathrm{~Hz}), 7.671(2 \mathrm{H}, \mathrm{t}, \mathrm{J}=7.6 \mathrm{~Hz}, 8 \mathrm{~Hz}), 8.378$ $(2 \mathrm{H}, \mathrm{d}, \mathrm{J}=7.6 \mathrm{~Hz})$.

5,6-Dibromo-1,10-phenanthroline, 3,5,6-tribromo-1,10phenanthroline, and 3,5,6,8-tetrabromo-1,10-phenanthroline were synthesized from 1,10-phenanthroline according to a literature procedure [49]. The $^{\circ}$ phenanthrolines ${ }^{\circ}$ were ${ }^{\circ}$ purified using successive recrystallizations. 5,6-Dibromo-1,10phenanthroline: ${ }^{1} \mathrm{H}$ NMR $\left(\mathrm{CDCl}_{3}\right) \delta=7.740(2 \mathrm{H}, \mathrm{dd}$, $\mathrm{J}=8.4 \mathrm{~Hz}, 8.0 \mathrm{~Hz}), 8.782(2 \mathrm{H}, \mathrm{dd}, \mathrm{J}=8.8 \mathrm{~Hz}, 1.2 \mathrm{~Hz})$, 9.226 (2H, dd, J = 4.4 Hz, $1.2 \mathrm{~Hz}) .3,5,6$-Tribromo-1,10phenanthroline: ${ }^{1} \mathrm{H}$ NMR $\left(\mathrm{CDCl}_{3}\right) \delta=7.748(1 \mathrm{H}, \mathrm{dd}$, $\mathrm{J}=8.8 \mathrm{~Hz}, 4.2 \mathrm{~Hz}), 8.765(1 \mathrm{H}, \mathrm{dd}, \mathrm{J}=8.8 \mathrm{~Hz}, 1.6 \mathrm{~Hz}), 8.928$ $(1 \mathrm{H}, \mathrm{d}, \mathrm{J}=2 \mathrm{~Hz}), 9.201$ (2H, m). 3,5,6,8-Tetrabromo-1,10phenanthroline: ${ }^{1} \mathrm{H}$ NMR $\left(\mathrm{CDCl}_{3}\right) \delta=8.920(2 \mathrm{H}, \mathrm{d}, \mathrm{J}=$ $2.2 \mathrm{~Hz}), 9.190(2 \mathrm{H}, \mathrm{d}, \mathrm{J}=2 \mathrm{~Hz})$.

A Varian $400 \mathrm{MHz}$ instrument (Varian Inc., Palo Alto, CA, USA) was used for all NMR spectroscopy.

\section{Metal Complexation/Mass Spectrometry}

Stock solutions of $4 \times 10^{-4} \mathrm{M}$ metal salts and auxiliary ligands were prepared in methanol. Solutions containing the flavonoid, metal salt, and auxiliary ligands were prepared in approximately 1:1:1 ratios at $1 \times 10^{-5} \mathrm{M}$.

The experiments were performed using a Thermo Finnigan (San Jose, CA, USA) LCQ Duo quadrupole ion trap (QIT) mass spectrometer equipped with an electrospray ionization (ESI) source. The flow rate of the solutions was $5 \mu \mathrm{l} \mathrm{min}{ }^{-1}$. The heated capillary temperature was $200^{\circ} \mathrm{C}$ and the ESI voltage was set at $+4.5 \mathrm{kV}$. The ionization times were set to $5-25 \mathrm{~ms}$ and each spectrum was an average of 20 scans. The lens and octapole voltages, sheath gas flow rate, and capillary voltage were optimized for maximum intensity of the ion corresponding to $[\mathrm{M}(\mathrm{II}) \text { (flavonoid }-\mathrm{H} \text { ) L }]^{+}$, where $\mathrm{M}$ is the metal and $\mathrm{L}$ is the auxiliary ligand. During MS/MS experiments, these parent ions were isolated and the CAD voltage was adjusted so that the parent ion intensity decreased to roughly $10 \%$ of the base peak. An isolation window sufficient to exclude all but the carbon-12 isotope was used for most experiments, and an isolation window sufficient to include all bromine isotopes was used for confirmation of the presence of the brominated ligands in selected fragment ions. 


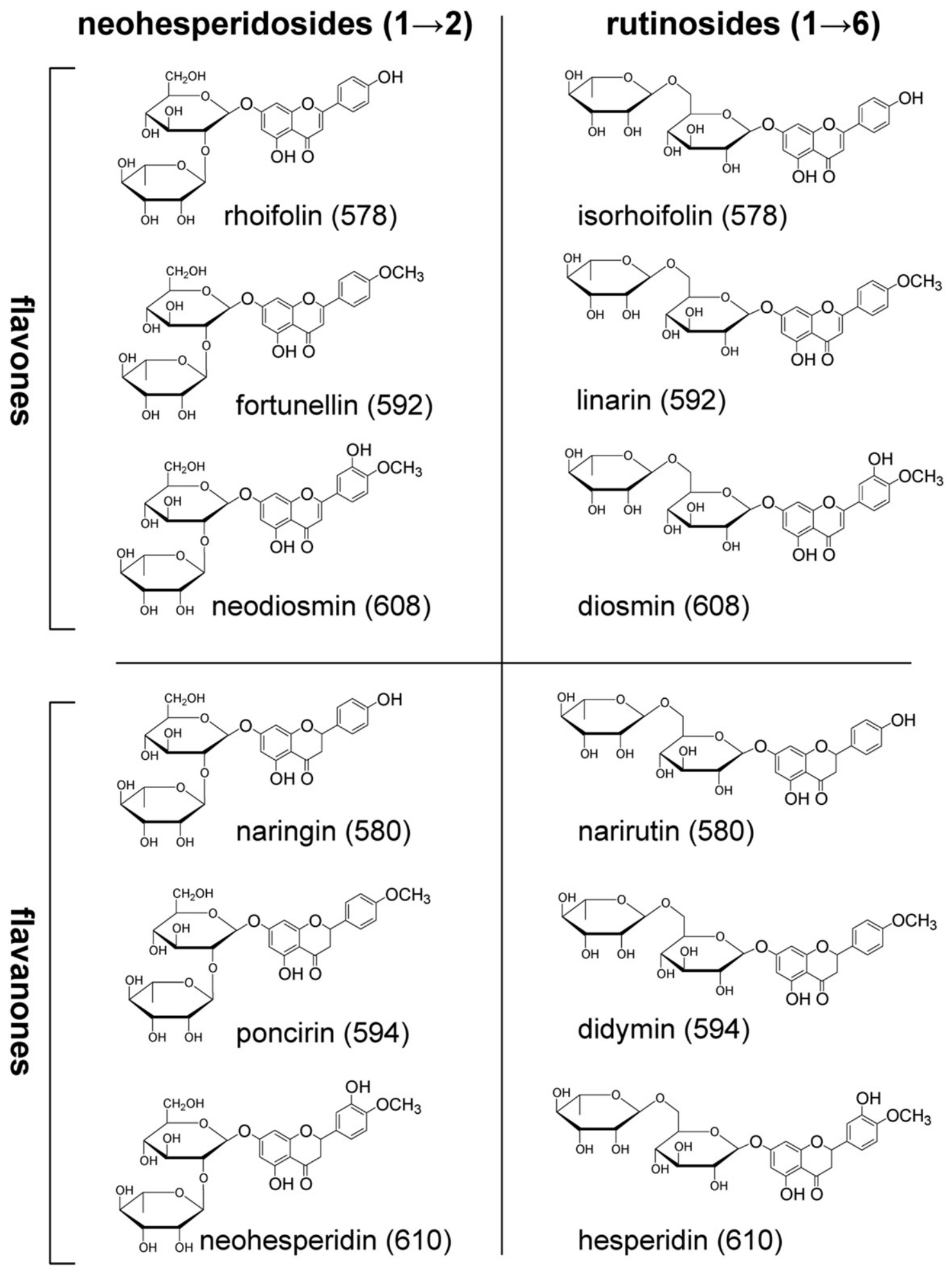

Figure 1. 7-O-Flavonoid diglycosides used in the study. Molecular weight is indicated in parentheses.

\section{Molecular Modeling}

Preoptimization and generation of coordinates was performed with CAChe Worksystem Pro using MM3/AM1. Final optimization was performed with the Gaussian'03 software package at the B3LYP/6-31G* level.

\section{Results and Discussion}

A series of six brominated pyridyl ligands were used in the ${ }^{\circ}$ present $^{\circ}$ study $^{\circ}\left(\right.$ Figure $\left.^{\circ} 2\right),{ }^{\circ}$ three ${ }^{\circ}$ based $^{\circ}$ on $^{\circ}$ the ${ }^{\circ} 2,2^{\circ}$ bipyridine skeleton (4,4'-dibromo-2,2' -bipyridine; $6,6^{\prime}$ dibromo-2,2'-bipyridine; and 4,4'-bis(bromomethyl)- 


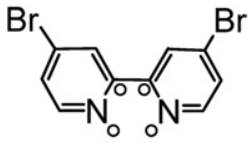

4,4'-dibromo2,2'-bipyridine

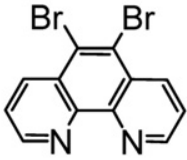

5,6-dibromo1,10-phenanthroline

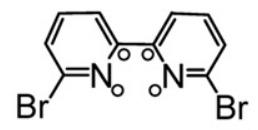

6,6'-dibromo2,2'-bipyridine<smiles></smiles>

3,5,6-tribromo1,10-phenanthroline

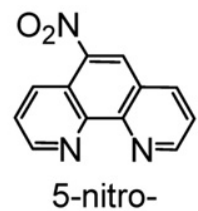

1,10-phenanthroline<smiles>BrCc1ccnc(-c2cc(CBr)ccn2)c1</smiles>

4,4'-bis(bromomethyl)2,2'-bipyridine<smiles>Brc1cnc2c(c1)c(Br)c(Br)c1cc(Br)cnc12</smiles>

3,5,6,8-tetrabromo1,10-phenanthroline

Figure 2. Ligands used in the study. The " $\bigcirc$ " symbols represent atoms discussed in the molecular modeling part of the study.

2,2'-bipyridine) and three based on the 1,10phenanthroline skeleton (5,6-dibromo-1,10-phenanthroline; 3,5,6-tribromo-1,10-phenanthroline; and 3,5,6,8-tetrabromo1,10-phenanthroline), with the overreaching goal of using them as "tunable" auxiliary ligands for complexation and structural characterization of isomers, as demonstrated specifically for flavonoids. Complexation with 4,4' - and 6,6'-dibromo-2,2' -bipyridine was compared to allow evaluation of the influence of the position of the electron-withdrawing groups. 4,4'-Bis(bromomethyl)2,2'-bipyridine was synthesized to investigate the effect of moving the bromine groups further from the aromatic ring. Three different brominated 1,10-phenanthroline compounds were synthesized to allow evaluation of the impact of the number of bromine groups on formation and dissociation of complexes. 5-Nitro-1,10-phenanthroline was used to evaluate the effect of an alternative electronwithdrawing substituent. For the experiments de- scribed in the following sections, ESI-MS/MS was used to analyze solutions containing either 2,2'-bipyridine or a brominated ligand in a solution containing one of 12 different flavonoids and either a copper or cobalt salt.

\section{Complexation with Bromobipyridines}

In the ESI mass spectra of each solution containing one flavonoid with 4,4'-dibromo-2,2' -bipyridine and either $\mathrm{CuBr}_{2}$ or $\mathrm{CoBr}_{2}$, the abundances of the resulting complexes are comparable. The spectra are dominated by the [M(II) (flavonoid - H) 4,4'-dibromo-2,2' -bipyridine $]^{+}$ complexes, without interference from the formation of numerous ${ }^{\circ}$ other $^{\circ}$ metal-ligand ${ }^{\circ}$ species $^{\circ}\left(\right.$ Figure $\left.^{\circ} 3\right) .{ }^{\circ}$ Division of the total ion current among several different metal-containing products has been one shortcoming noted in some previous metal complexation studies,

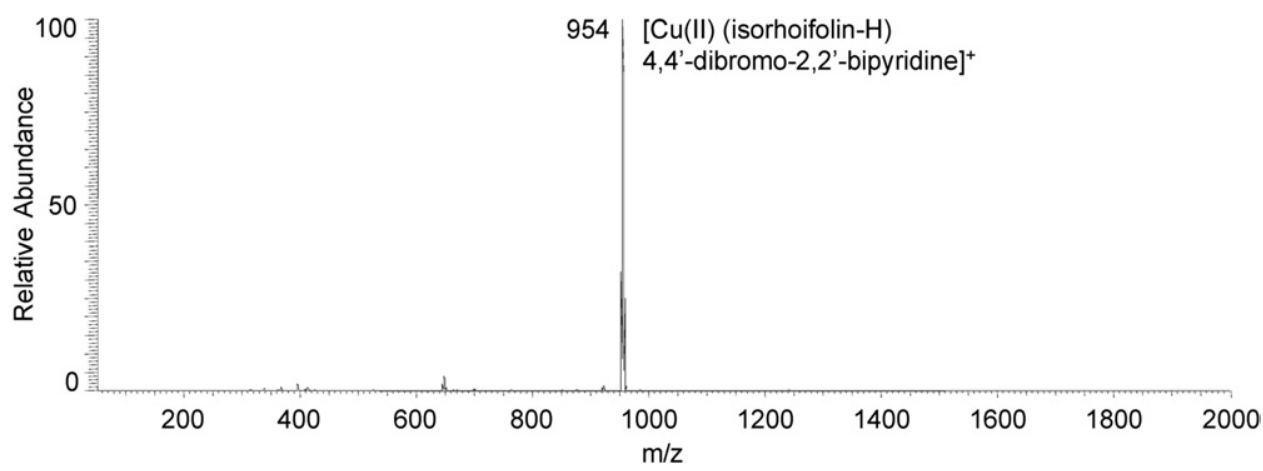

Figure 3. Full-scan spectrum of a solution of rhoifolin $/ \mathrm{Cu}(\mathrm{II}) / 4,4^{\prime}$-dibromo-2,2'-bipyridine showing the flavonoid/metal/ligand complex as the dominant species. 

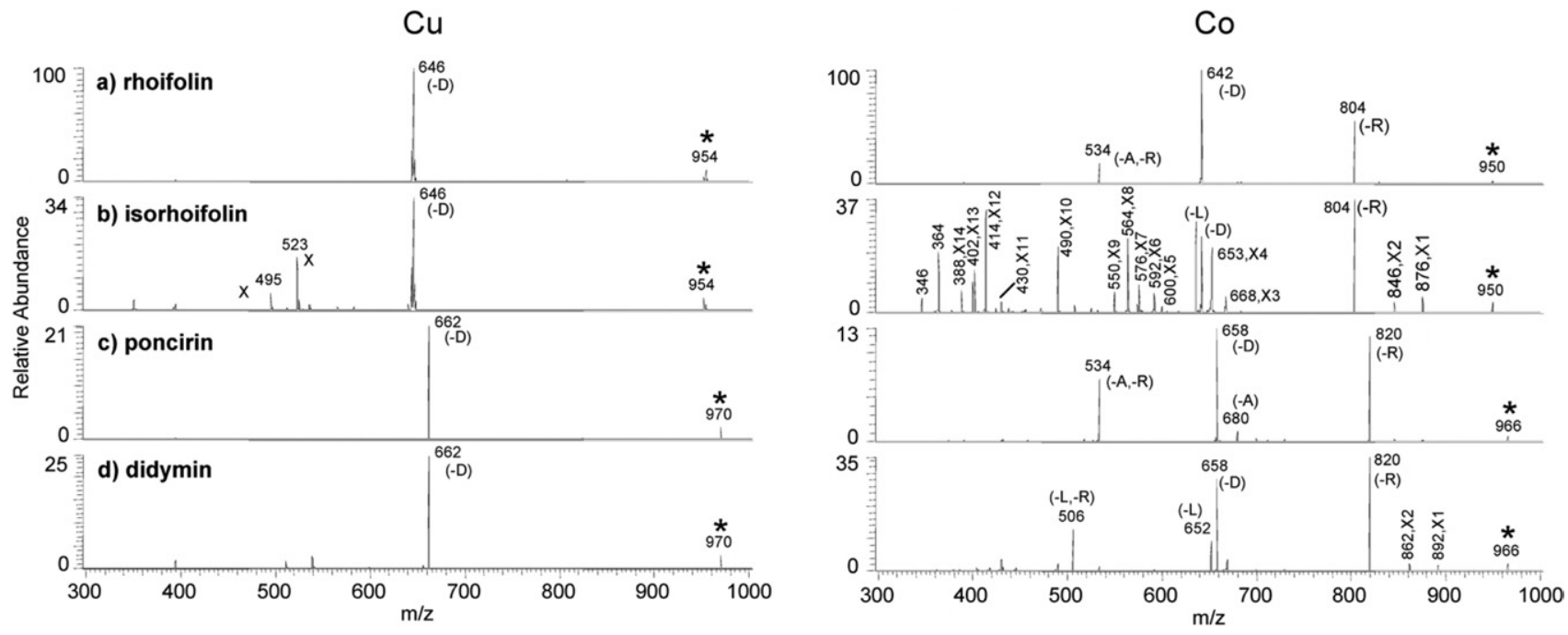

Figure 4. Representative spectra for CAD of flavonoid/metal $/ 4,4^{\prime}$-dibromo-2,2'-bipyridine with a flavone rutinoside/neohesperidoside and a flavanone rutinoside/neohesperidoside, comparing the $\mathrm{Cu}$ and $\mathrm{Co}$ complexes. (-D) indicates the loss of disaccharide moiety, (-R) indicates the loss of rhamnose moiety, (-L) indicates the loss of ligand, and X indicates a cross-ring cleavage of the sugar moiety.

thus having a negative impact on ultimate detection limits.

Because both copper and cobalt resulted in the formation of equally abundant flavonoid complexes, the CAD spectra were acquired for all the complexes of the ${ }^{\circ}$ flavonoids ${ }^{\circ}$ used $^{\circ}$ in $^{\circ}$ the ${ }^{\circ}$ study. ${ }^{\circ}$ Figure ${ }^{\circ} 4^{\circ}$ shows ${ }^{\circ}$ this comparison for one representative flavonoid from each of ${ }^{\circ}$ the ${ }^{\circ}$ four ${ }^{\circ}$ classes $^{\circ}$ summarized ${ }^{\circ}{ }^{\circ}{ }^{\circ}$ Figure $^{\circ} 1^{\circ}$ (that ${ }^{\circ}$ is, ${ }^{\circ}$ one flavone neohesperidoside, one flavone rutinoside, one flavanone neohesperidoside, and one flavanone rutinoside). All of the copper complexes dissociate predominantly by the loss of the disaccharide moiety, corresponding to the neutral loss of 308 Da (left panel of Figure $\left.{ }^{\circ} 4\right)^{\circ}{ }^{\circ}$ The $^{\circ}$ copper $^{\circ}$ complex $^{\circ}$ of ${ }^{\circ}$ isorhoifolin ${ }^{\circ}$ exhibits two additional fragment ions arising from cross-ring cleavages of the disaccharide (cross-ring cleavages are designated by $\mathrm{X}$ in the figures) in conjunction with loss of the 4,4'-dibromo-2,2'-bipyridine ligand, yielding the fragment ions at $m / z 523$ and 495 . The cobalt complexes exhibit more intricate fragmentation pathways with unique ${ }^{\circ}$ ions ${ }^{\circ}$ for ${ }^{\circ}$ each $^{\circ}$ flavonoid ${ }^{\circ}$ (right ${ }^{\circ}$ panel ${ }^{\circ}$ of ${ }^{\circ}$ Figure ${ }^{\circ}$ ). The most common ones include the loss of the terminal rhamnose group $(-146 \mathrm{Da})$, loss of the disaccharide moiety (-308 Da), loss of the auxiliary 4,4'-dibromo-2,2' bipyridine ligand $(-314 \mathrm{Da})$, loss of the aglycon group $(-\mathrm{A})$, and other combined losses thereof. There is at least one diagnostic fragment ion for each of the isomers, and in most cases there are at least two. In addition, there are two diagnostic cross-ring cleavages involving the disaccharide group for each of the flavone and flavanone rutinosides (that is, isorhoifolin and didymin in Figure $\left.^{\circ} 4\right) .^{\circ}$ The $^{\circ}$ resulting ${ }^{\circ}$ ions $^{\circ}$ are $^{\circ}$ observed $^{\circ}$ at ${ }^{\circ} \mathrm{m} / z 876$ $(-74, \mathrm{X} 1)$ and $\mathrm{m} / \mathrm{z} 846(-104, \mathrm{X} 2)$ for the isorhoifolin complex and $m / z 892(-74, \mathrm{X} 1)$ and $m / z 862(-104, \mathrm{X} 2)$ for the didymin complex. Proposed cleavages that are consistent with the observed fragment ions are shown in Scheme $\mathbf{1}$ for these cross-ring cleavages. There is another notable series of cross-ring cleavages for the flavone rutinoside isomers (fragment ions labeled $\mathrm{XN}$ in the 9 right ${ }^{9}$ anel ${ }^{\circ}$ of Figure 3 B for the isorhoifolin ${ }^{\circ}$ complex, also seen for the analogous complexes of diosmin and linarin). Some of these cross-ring cleavages occur in conjunction with loss of the 4,4'-dibromo-2,2'-bipyridine ligand.

It is interesting that these extensive cross-ring fragmentations are observed for the flavone rutinoside complexes but not the flavanone rutinoside complexes. We speculate that this striking difference in the degree of cross-ring cleavages is related to the ability of the rutinose moiety to interact with the metal center in the complexes, which may influence the migration of the metal or auxiliary ligand chelate upon activation, as well as the strength of the glycosidic bond. Previous

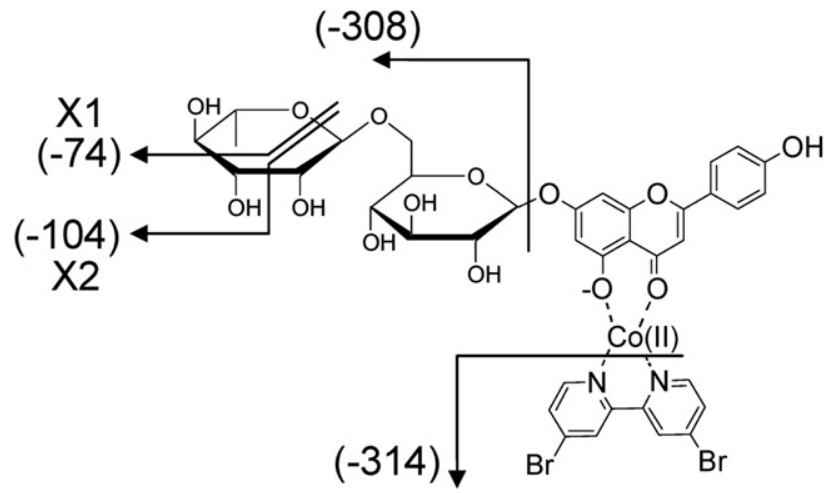

Scheme 1. Proposed cleavages for a flavonoid/Co/4,4'dibromo-2,2'-bipyridine cobalt complex using isorhoifolin as an example. 
computational modeling of [Co(II) (flavonoid - H) $\left(2,2^{\prime}\right.$-bipyridine $\left.)\right]^{+^{\circ}}$ complexes $^{\circ}$ by $^{\circ}$ Zhang $^{\circ}$ and $^{\circ}$ Brodbelt [50] revealed that the most 9 favorable metal ${ }^{\circ}$ coordination sites involved the carbonyl oxygen atom and adjacent deprotonated oxygen atom of the aglycon. Moreover, it was shown that for the rutinosides, the disaccharide folds toward the metal center, thus increasing the interaction ${ }^{\circ}$ of ${ }^{\circ}$ the ${ }^{\circ}$ disaccharide ${ }^{\circ}$ with ${ }^{\circ}$ the ${ }^{\circ}$ metal ${ }^{\circ}[50]$. This factor presumably could enhance the possibility of migration of the metal/auxiliary ligand chelate in the complexes containing the 4,4'-dibromo-2,2'-bipyridine ligands and/or modify the electron density in the glycoside bonds, thus promoting cross-ring cleavages instead of glycosidic bond cleavage. It was also found that the glycosidic bond energies and point charges for the aglycon-disaccharide bonds were greater for the flavonoid rutinosides than for the flavonoid neohesperidosides 950$]$. This ${ }^{\circ}$ effect ${ }^{\circ}$ may ${ }^{\circ}{ }^{\circ}$ further ${ }^{\circ}$ exaggerated for the complexes containing the 4,4'-dibromo-2,2'bipyridine ligand, given that the electron-withdrawing bromine atoms should temper the metal binding energy of the auxiliary ligand, which could translate into alternate fragmentation pathways to glycosidic bond cleavage, such as cross-ring cleavages.

The analytical utility of two other brominated bipyridine ligands, 6,6'-dibromo-2,2'-bipyridine and 4,4'bis(bromomethyl)-2,2'-bipyridine, was also evaluated. The only complexes observed by ESI-MS for solutions containing a flavonoid, a metal salt, and 6,6'-dibromo$2,2^{\prime}$-bipyridine were ligand/metal complexes of the type [M(I) 6,6'-dibromo-2,2'-bipyridine $\left.+\mathrm{H}_{2} \mathrm{O}\right]^{+}$. Molecular modeling was used to evaluate the 4,4'dibromo-2,2'-bipyridine/Co(II) and 6,6'-dibromo-2,2'bipyridine/Co(II) complexes to assess the potential hindrance to flavonoid binding. The dihedral angles of the $\mathrm{N}-\mathrm{C}-\mathrm{C}-\mathrm{N}$ bonds on the ligand (labeled with a " $O$ " In Figure?) ${ }^{\circ}$ were found to have ${ }^{\circ}$ aminimum ${ }^{\circ}$ energy at $0^{\circ}$ for both complexes, and this is consistent with previous modeling studies of other flavonoid metal complexes ${ }^{\circ}$ containing $^{\circ}$ pyridy $l^{\circ}$ ligands ${ }^{\circ}[50]{ }^{\circ}{ }^{\circ} \mathrm{The}{ }^{\circ} \mathrm{C}-\mathrm{Br}$ bond distances were $1.9248 \AA$ for the 6,6'-dibromo-2,2'bipyridine complex. These distances, coupled with the dihedral angle that shows that the bromine atoms are in the same plane with $\mathrm{Co}$, indicate that the proximity of the bromine atoms to the cobalt ion causes substantial steric hindrance, which prevents simultaneous coordination of 6,6'-dibromo-2,2'-bipyridine and a flavonoid to the metal ion, thus making 6,6'-dibromo-2,2'-bipyridine a poor auxiliary ligand.

In contrast to the ESI-MS results for the 6,6'-dibromo2,2-bipridine/flavonoid/metal solutions, abundant flavonoid complexes were observed for solutions containing 4,4'-bis(bromomethyl)-2,2'-bipyridine and a transition metal. However, the CAD spectra were dominated by the loss of the rhamnose moiety and the loss of the disaccharide group. These CAD spectra did not allow differentiation of the various isomers, and thus

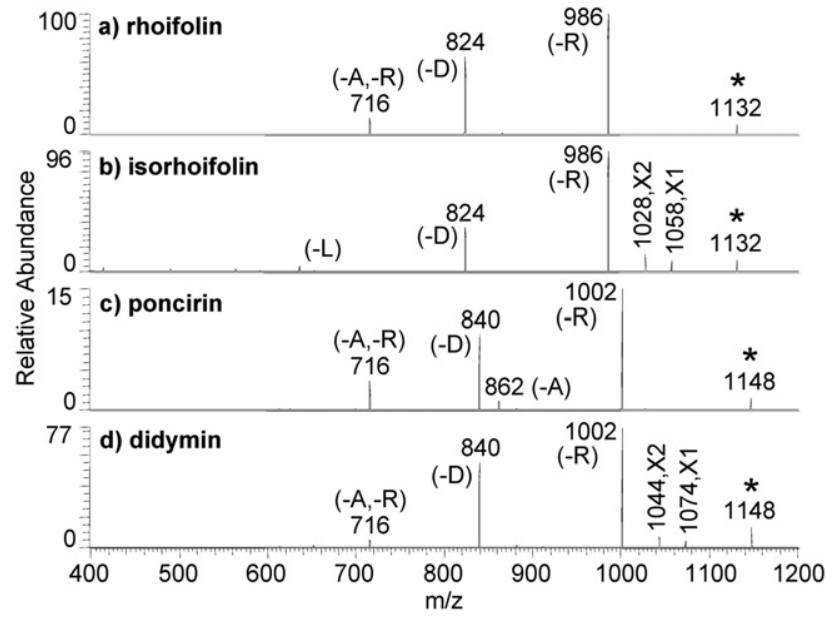

Figure 5. Representative CAD mass spectra of the $[3,5,6,8-$ tetrabromo-1,10-phenanthroline (flavonoid - H) $\mathrm{Co}(\mathrm{II})]^{+}$complexes containing the following flavonoids: (a) rhoifolin, (b) isorhoifolin, (c) poncirin, and (d) didymin. (-D) indicates the loss of disaccharide moiety, (-R) indicates the loss of rhamnose moiety, and (-A) indicates the loss of aglycon.

4,4'-bis(bromomethyl)-2,2'-bipyridine proved to be an unsatisfactory auxiliary ligand.

\section{Complexation with Phenanthroline Ligands}

The complexation of each of the bromophenanthrolines (structures ${ }^{\circ}$ shown ${ }^{\circ}{ }^{\circ}{ }^{\circ}$ Figure 2$)^{\circ}{ }^{\circ}$ as $^{\circ}$ studied ${ }^{\circ}$ with $^{\circ}$ each ${ }^{\circ}$ of the ${ }^{\circ} 12^{\circ}$ flavonoids. ${ }^{\circ}$ Figure $^{\circ} 5^{\circ}$ illustrates ${ }^{\circ}$ the ${ }^{\circ} \mathrm{CAD}^{\circ}$ mass spectra of the complexes containing the 3,5,6,8tetrabromo-1,10-phenanthroline ligand, four representative flavonoids, and cobalt. The dominant dissociation route ${ }^{\circ}$ for ${ }^{\circ}$ all ${ }^{\circ}$ four ${ }^{\circ}$ flavonoid ${ }^{\circ}$ complexes $^{\circ}$ in ${ }^{\circ} F_{i g u r e}{ }^{\circ}{ }^{\circ}$ is the loss of the rhamnose moiety, as well as elimination of the disaccharide group, two pathways that are commonly observed for other types of flavonoid/metal complexes. The presence of other unique fragment ions in each CAD spectrum allow each pair of isomers to be readily distinguished.

The types of fragment ions observed for all of the cobalt complexes containing each of the bromophenanthroline ligands as compared to unmodified 1,10phenanthroline and each of the flavonoids are summarized ${ }^{\circ}$ in $^{\circ} \mathrm{Table}^{\circ}{ }^{\circ} .{ }^{\circ} \mathrm{In}^{\circ}$ general, ${ }^{\circ}$ the ${ }^{\circ}$ type ${ }^{\circ}$ of ${ }^{\circ}$ fragmentation pathways are similar to those noted earlier for the complexes containing the 4,4'-dibromo-2,2'-bipyridine ligand, including the loss of the rhamnose group, the elimination of the disaccharide moiety, and the loss of the aglycon and rhamnose groups. The most notable difference between the fragmentation patterns of the flavonoid rutinosides and the neohesperidosides are two cross-ring cleavages $(-74 \mathrm{Da},-104 \mathrm{Da})$ that were also observed for the analogous flavonoid rutinoside complexes containing the 4,4'-dibromo-2,2'-bipyridine ligand. In addition, the flavanone neohesperidosides are differentiated from the flavones by the characteristic loss of the aglycon moiety. 
Table 1. Summary of fragment ion distributions observed in the CAD spectra for selected Co/flavonoid/ligand complexes grouped by flavonoid diglycoside class ${ }^{\mathrm{a}}$

\begin{tabular}{|c|c|c|c|c|c|c|c|}
\hline 4,4'-dibromo-2,2'-bipyridine/Co & $(-R)$ & $(-\mathrm{D})$ & $(-A)$ & $(-A,-R)$ & $(-L)$ & $\mathrm{X} 1$ & $\mathrm{X} 2$ \\
\hline Flavone neohesperidoside (rhoifolin) & 54 & 100 & & 19 & & & \\
\hline Flavone rutinoside (isorhoifolin) & 100 & 68 & & & 80 & 10 & 14 \\
\hline Flavanone neohesperidoside (poncirin) & 95 & 100 & 8 & 58 & & & \\
\hline Flavanone rutinoside (didymin) & 100 & 83 & & & 29 & 5 & 7 \\
\hline 1,10-phenanthroline/Co & $(-R)$ & $(-D)$ & $(-A)$ & $(-A,-R)$ & $(-\mathrm{L})$ & $\mathrm{X} 1$ & $\mathrm{X} 2$ \\
\hline Flavone neohesperidoside (rhoifolin) & 15 & 100 & & 6 & & & \\
\hline Flavone rutinoside (isorhoifolin) & 14 & 100 & & & & & 4 \\
\hline Flavanone neohesperidoside (poncirin) & 38 & 100 & & 32 & & & \\
\hline Flavanone rutinoside (didymin) & 16 & 100 & & 8 & & & \\
\hline 5,6,-Dibromo-1,10-phenanthroline/Co & $(-R)$ & $(-D)$ & $(-A)$ & $(-A,-R)$ & $(-L)$ & $\mathrm{X} 1$ & $\mathrm{X} 2$ \\
\hline Flavone neohesperidoside (rhoifolin) & 73 & 100 & & 23 & & & \\
\hline Flavone rutinoside (isorhoifolin) & 94 & 100 & & & & 15 & 21 \\
\hline Flavanone neohesperidoside (poncirin) & & 100 & & & & & \\
\hline Flavanone rutinoside (didymin) & & 100 & & & & & \\
\hline 3,5,6-Tribromo-1,10-phenanthroline/Co & $(-R)$ & $(-D)$ & $(-A)$ & $(-A,-R)$ & $(-L)$ & $\mathrm{X} 1$ & $\mathrm{X} 2$ \\
\hline Flavone neohesperidoside (rhoifolin) & 100 & 89 & & 15 & & & \\
\hline Flavone rutinoside (isorhoifolin) & 100 & 61 & & 4 & & 12 & 18 \\
\hline Flavanone neohesperidoside (poncirin) & 79 & 100 & 5 & 26 & & & \\
\hline Flavanone rutinoside (didymin) & 94 & 100 & & 8 & & 4 & 11 \\
\hline 3,5,6,8-Tetrabromo-1,10-phenanthroline/Co & $(-R)$ & $(-D)$ & $(-A)$ & $(-A,-R)$ & $(-L)$ & $\mathrm{X} 1$ & $\mathrm{X} 2$ \\
\hline Flavone neohesperidoside (rhoifolin) & 100 & 68 & & 14 & & & \\
\hline Flavone rutinoside (isorhoifolin) & 100 & 36 & & & 5 & 8 & 14 \\
\hline Flavanone neohesperidoside (poncirin) & 100 & 56 & 8 & 20 & & & \\
\hline Flavanone rutinoside (didymin) & 100 & 72 & & 6 & & 11 & 6 \\
\hline 5-Nitro-1,10-phenanthroline/Co & $(-R)$ & $(-D)$ & $(-A)$ & $(-A,-R)$ & $(-L)$ & $\mathrm{X} 1$ & $\mathrm{x} 2$ \\
\hline Flavone neohesperidoside (rhoifolin) & 38 & 100 & & 14 & & & \\
\hline Flavone rutinoside (isorhoifolin) & 31 & 100 & & & 8 & 6 & 12 \\
\hline Flavanone neohesperidoside (poncirin) & 100 & 100 & & 66 & & & \\
\hline Flavanone rutinoside (didymin) & 48 & 100 & & & 10 & 6 & 14 \\
\hline
\end{tabular}

a(-D) indicates loss of disaccharide moiety; (-R) indicates loss of rhamnose moiety; (-L) indicates loss of ligand; (-A) indicates loss of aglycon; and $X 1$ and $X 2$ represent the cross-ring cleavages of $(-74)$ and $(-104)$, respectively $( \pm 2 \%)$.

The summary in Table'19reveals that the number of different fragment ions increases as the number of electron-withdrawing bromine atoms on the 1,10phenanthroline auxiliary ligand increases. The number of fragmentation pathways correlates with the relative stabilities of the metal complexes. Complexes in which one of the two coordinated molecules (that is, flavonoid versus auxiliary ligand) has a significantly greater metal binding energy than that of the other tend to dissociate by just a few routes, thus typically being less useful for confident differentiation of isomers. Among the three brominated phenanthroline ligands, the 3,5,6,8-tetrabromo-1,10-phenanthroline ligand is expected to have the lowest metal binding energy as a result of the influence of the four electron-withdrawing bromine atoms on the metalcoordinating nitrogen atoms. This makes the metal binding energy of the 3,5,6,8-tetrabromo-1,10phenanthroline ligand closer to the metal binding energy of a typical flavonoid, thus increasing the stability of the 3,5,6,8-tetrabromo-1,10-phenanthroline/flavonoid complexes and resulting in a greater array of competitive dissociation routes. In fact, of the three bromophenanthroline ligands, the tetrabromo ligand (3,5,6,8-tetrabromophenanthroline) is the only one that allows successful differentiation of all of the flavonoid isomers, in terms of providing at least one unique diagnostic fragment ion for each isomer.

To further assess the impact of electron-withdrawing groups on the tunability of the auxiliary ligands, 5-nitro1,10-phenanthroline was evaluated because the nitro group is one of the most electron-withdrawing groups available. Abundant complexes of the type [M(II) (flavonoid - H) 5-nitro-1,10-phenanthroline $]^{+}$were formed with the flavonoids, and the resulting CAD spectra also displayed the enhanced diagnostic cross-ring cleavages observed ${ }^{\circ}$ with $^{\circ}$ the ${ }^{\circ}$ brominated $^{\circ}$ ligands $^{\circ}\left(\right.$ Table $\left.^{\circ} 1\right){ }^{\circ}{ }^{\circ}$ This result further underscores the strategy of adding electron-withdrawing groups to alter the dissociation pathways of the targeted complexes. 


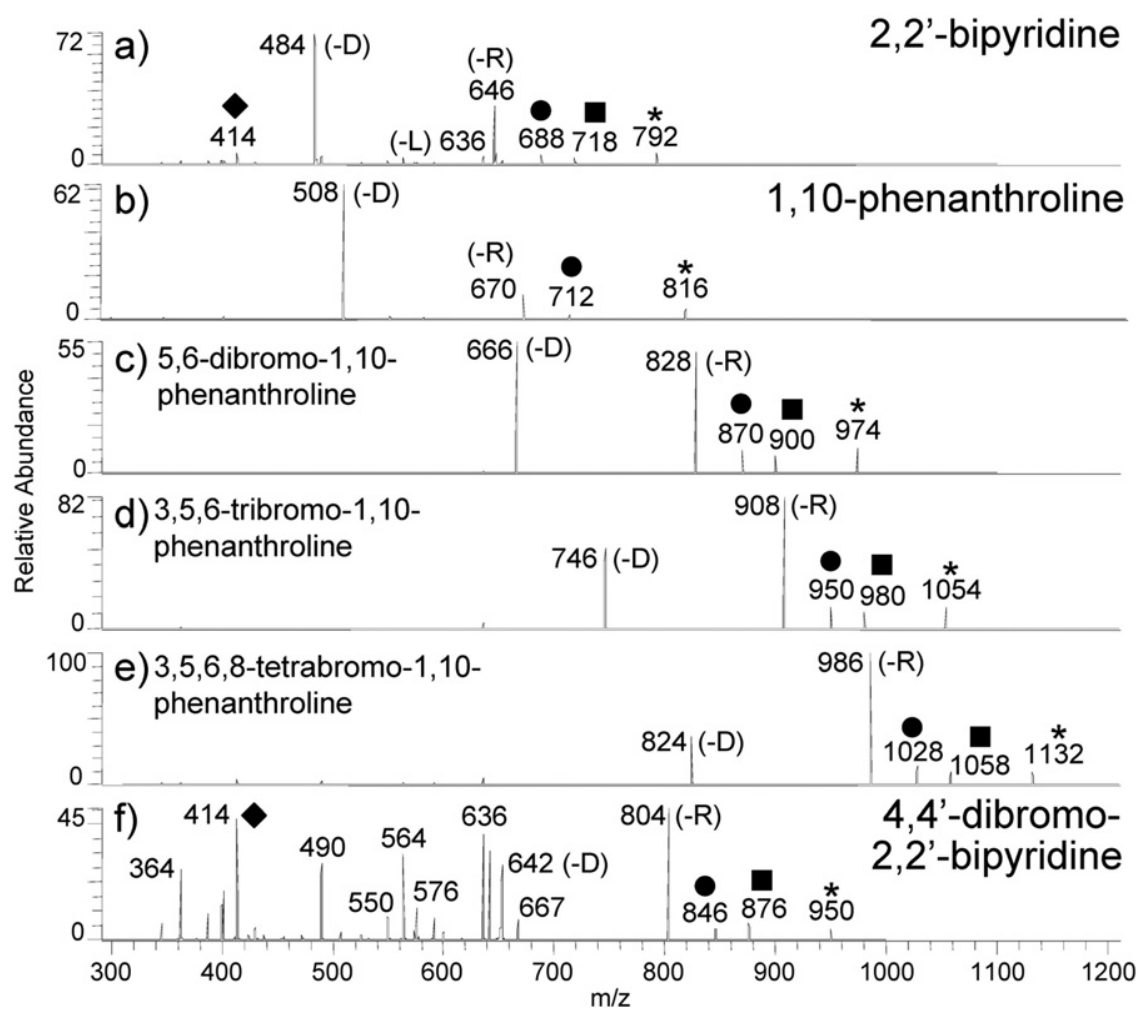

Figure 6. Comparison of the CAD mass spectra of $[\mathrm{Co}(\mathrm{II}) \text { (isorhoifolin-H) } \mathrm{L}]^{+}$complexes containing each brominated ligand, where $\mathrm{L}$ is the brominated ligand. A square and a circle are used to represent the cross-ring cleavages resulting in losses of 74 and $104 \mathrm{Da}$, respectively. A diamond is used to represent loss of the ligand in conjunction with a cross-ring cleavage of the sugar. (-D) indicates the loss of disaccharide moiety, (-R) indicates the loss of rhamnose moiety, (-L) indicates the loss of ligand, and $\mathrm{X}$ indicates a cross-ring cleavage of the sugar moiety.

\section{Comparison of All Ligands}

Figure ${ }^{\circ} 6^{\circ}$ shows $^{\circ}$ the ${ }^{\circ} \mathrm{CAD}^{\circ}$ mass $^{\circ}$ spectra ${ }^{\circ}$ for ${ }^{\circ} \mathrm{a}^{\circ}$ series $^{\circ}$ of cobalt/isorhoifolin complexes, each containing one of six $^{\circ}$ different ${ }^{\circ}$ auxiliary ${ }^{\circ}$ ligands. ${ }^{\circ}$ Figure ${ }^{\circ} 6^{\circ}$ comprehensively ${ }^{\circ}$ illustrates ${ }^{\circ}$ how $^{\circ}$ the ${ }^{\circ}$ bromination ${ }^{\circ}$ of $^{\circ}$ the ${ }^{\circ}$ auxiliary ligands affects the tunability of the CAD patterns. The $\mathrm{CAD}$ mass spectra for the complexes containing either 2,2'-bipyridine or 1,10-phenanthroline are also shown for comparison. A quick inspection of the CAD mass spectra reveals notable differences in the relative abundances of specific fragment ions, in addition to the emergence of new diagnostic fragment ions as the identity of the auxiliary ligand changes. For all of the complexes, the elimination of the rhamnose moiety (-R) and the loss of the disaccharide group (-D) is consistently observed, and the relative abundances of the two resulting fragment ions vary significantly for the series of $\mathrm{CAD}$ spectra. As the degree of bromination increases for the phenanthroline ligands, the ratio of $(-R) /(-D)$ increases $^{\circ}\left(\right.$ Figure $\left.^{\circ} 6 \mathrm{c}-\mathrm{Q}\right) .{ }^{\circ}$ Another ${ }^{\circ}$ notable ${ }^{\circ}$ difference ${ }^{\circ}$ is $^{\circ}$ the increase in the abundance of the two cross-ring cleavages X1 (-74, labeled with a square) and X2 (-104, labeled with a circle) upon bromination of the auxiliary ligands. Also noteworthy is the increase in the abundance ${ }^{\circ}$ of ${ }^{\circ}$ the ${ }^{\circ}$ fragment ${ }^{\circ}$ ion ${ }^{\circ}$ of ${ }^{\circ} 414^{\circ} \mathrm{Da}^{\circ}$ (Figure 6 ff, ${ }^{\circ}$ labeled with a diamond) for the complex containing 4,4'- dibromo-2,2'-bipyridine, which results from the loss of the ligand in conjunction with a cross-ring cleavage of the sugar. Although the CAD spectra of complexes containing 2,2'-bipyridine or 1,10-phenanthroline yielded some of the same diagnostic fragments, there was an enhancement in the abundance of these fragment ions using the brominated ligands.

Overall, Figure ${ }^{\circ}{ }^{\circ}$ and ${ }^{\circ}$ Table ${ }^{\circ} 1{ }^{\circ}$ clearly $^{\circ}$ demonstrate the tunability of the CAD patterns promoted by the use of different auxiliary ligands. This tunability is related to the relative metal binding energies of the auxiliary ligand and flavonoids. Based on the significant differences in fragmentation pathways of pairs of isomeric complexes ${ }^{\circ}$ observed ${ }^{\circ}$ earlier ${ }^{\circ}$ in ${ }^{\circ}$ Figure $44^{\circ}$ and ${ }^{\circ}$ compared to the performance of the other auxiliary ligands, 4,4' dibromo-2,2'-bipyridine was judged to be the best overall auxiliary ligand for the differentiation of the series of flavonoids in the present study.

\section{Electron-Withdrawing Group Effect}

The results presented in this work show that the tunability of dissociation of flavonoid complexes is accomplished by adding electron-withdrawing substituents to the auxiliary ligands used to coordinate the transition metal and stabilize the flavonoid complexes. To under- 
stand the impact of the electron-withdrawing substituents on the stabilities and dissociation pathways of the flavonoid complexes in the gas phase, it is instructive to consider the influence of electron-withdrawing substituents on the binding energies of pyridyl ligands in solution. Although extensive solution data are not available, numerous binding constants have been measured for pyridyl ligands with $\mathrm{Co}(\mathrm{II})$ and $\mathrm{Fe}(\mathrm{II})$, two representative transition metals, in aqueous solution. $\mathrm{Fe}(\mathrm{II})$ was not a metal used in the present ESI-MS study, although the binding constants for $\mathrm{Fe}(\mathrm{II})$ complexes were compiled because this is the only metal for which brominated ligands have been studied.

The binding constants for cobalt/pyridyl ligand complexes in solution decrease in the order: 5,6-dimethyl1,10-phenanthroline $(\log \mathrm{K}$ 7.47) > 5-methyl-1,10phenanthroline $(\log \mathrm{K} 7.14)>1,10$-phenanthroline (log $\left.\mathrm{K}^{\circ} 7.08\right)^{\circ}>{ }^{\circ} 5$-nitrophenanthroline ${ }^{\circ}\left(\log ^{\circ} \mathrm{K}^{\circ} 6.3\right)^{\circ}[43]^{\circ},{ }^{\circ}$ thus showing the impact of the addition of electron-releasing versus electron-withdrawing substituents on 1,10phenanthroline. The binding constants for $\mathrm{Fe}(\mathrm{II}) /$ pyridyl complexes decrease in the order: 5,6-dimethyl1,10-phenanthroline $(\log \mathrm{K}$ 6.37) > 5-methyl-1,10phenanthroline $(\log K$ 6.00) $>$ 1,10-phenanthroline $(\log \mathrm{K} 5.85)>$ 5-bromo-1,10-phenanthroline (log K 5.65) > 5-nitrophenanthroline ${ }^{\circ}\left(\log \mathrm{K}^{\circ} 5.06\right)^{\circ}[43]^{\circ}{ }^{\circ}$ This ${ }^{\circ}$ trend indicates that the metal/ligand binding energies decrease as the substituents on the ligand become more electron withdrawing. A similar inductive effect would be expected for the metal/ligand binding energies in the gas phase. The variations in the metal/ligand binding energies may influence the energetics of dissociation pathways and also affect the preferred coordination sites of the flavonoid complexes. The ligand also has an impact on the way the disaccharide moiety of the flavonoid interacts with the metal. As mentioned previously, modeling studies have indicated that for the rutinoside isomers, the disaccharide folds toward the metal center [50]..$^{\circ}$ The $^{\circ}$ addition $^{\circ}$ of $^{\circ}$ bromo $^{\circ}$ substituents $^{\circ}$ changes $^{\circ}$ the steric environment of the auxiliary ligands, which may influence the conformations of the complexes and thus the degree of interaction of the disaccharide group with the ${ }^{\circ}$ metal $^{\circ}$ center $^{\circ}[50]$.

\section{Conclusions}

The fragmentation pathways of flavonoids can be tuned by using a metal complexation strategy that incorporates auxiliary ligands containing electron-withdrawing substituents. Upon collisional activation, the resulting $\left[\mathrm{Co}(\mathrm{II})\right.$ (flavonoid $-\mathrm{H}$ ) auxiliary ligand ${ }^{+}$complexes dissociate by pathways that are characteristic of flavone and flavanone 7-O-diglycosides (that is, loss of rhamnose and disaccharide groups), in addition to those that allow differentiation of isomers that differ only in the type of intersaccharide linkage. In this work, the pathways that distinguish flavone and flavanone disaccharides with $1 \rightarrow 2$ and $1 \rightarrow 6$ linkages have been enhanced by adding electron-withdrawing substituents to the auxiliary ligands. The addition of electronwithdrawing groups to the auxiliary ligands reduces their metal-ligand binding affinities and consequently alters the stabilities and conformations of the [M(II) (flavonoid $-\mathrm{H}) \mathrm{LJ}^{+}$complexes, thus resulting in the enhancement of diagnostic fragmentation pathways.

\section{Acknowledgments}

Funding from the Welch Foundation (F1155) and the National Science Foundation (CHE-0315337) is gratefully acknowledged. We thank Joe Chipuk for assistance with the molecular modeling.

\section{References}

1. Reddy, P. N.; Ramesh, V.; Srinivas, R.; Sharma, G. V. M.; Nagendar, P.; Subash, V. Differentiation of Some Positional and Diastereomeric Isomers of Boc-carbo-beta(3) Dipeptides Containing Galactose, Xylose and Mannose Sugars by Electrospray Ionization Tandem Mass Spectrometry (ESI MS/MS). Int. J. Mass Spectrom. 2006, 248, 115-123.

2. Song, F. R.; Liu, Z. Q.; Liu, S. Y.; Cai, Z. W. Differentiation and Identification of Ginsenoside Isomers by Electrospray Ionization Tandem Mass Spectrometry. Anal. Chim. Acta 2005, 531, 69-77.

3. Wu, L. M.; Cooks, G. Chiral and Isomeric Analysis by Electrospray Ionization and Sonic Spray Ionization Using the Fixed-ligand Kinetic Method. Eur. J. Mass Spectrom. 2005, 11, 231-242.

4. Song, F. R.; Cui, M.; Liu, Z. Q.; Yu, B.; Liu, S. Y. Multiple-stage Tandem Mass Spectrometry for Differentiation of Isomeric Saponins. Rapid Commun. Mass Spectrom. 2004, 18, 2241-2248.

5. Matamoros, F. L. E.; Obel, N.; Scheller, H. C.; Roepstorff, P. Differentiation of Isomeric Oligosaccharide Structures by ESI Tandem MS and GC-MS. Carbohydr. Res. 2004, 339, 655-664.

6. Zhang, J. H.; Lindsay, L. L.; Hedrick, J. L.; Lebrilla, C. B. Strategy for Profiling and Structure Elucidation of Mucin-type Oligosaccharides by Mass Spectrometry. Anal. Chem. 2004, 76, 5990-6001.

7. Cuyckens, F.; Rozenberg, R.; de Hoffmann, E.; Claeys, M. Structure Characterization of Flavonoid O-Diglycosides by Positive and Negative Nano-electrospray Ionization Ion Trap Mass Spectrometry. J. Mass Spectrom. 2001, 36, 1203-1210.

8. Desaire, H.; Leary, J. A. Multicomponent Quantification of Diastereomeric Hexosamine Monosaccharides Using Ion Trap Tandem Mass Spectrometry. Anal. Chem. 1999, 71, 4142-4147.

9. Waridel, P.; Wolfender, J. L.; Ndjoko, K.; Hobby, K. R.; Major, H. J. Hostettmann, K. Evaluation of Quadrupole Time-of-Flight Tandem Mass Spectrometry and Ion-Trap Multiple-stage Mass Spectrometry for the Differentiation of C-glycosidic Flavonoid Isomers. J. Chromatogr. A 2001, 926, 29-41.

10. Gaucher, S. P.; Leary, J. A. Influence of Metal Ion and Coordination Geometry on the Gas Phase Dissociation and Stereochemical Differentiation of N-glycosides. Int. J. Mass Spectrom. 2000, 197, 139-148.

11. Desaire, H.; Leary, J. A. Differentiation of Diastereomeric N-acetylhexosamine Monosaccharides Using Ion Trap Tandem Mass Spectrometry. Anal. Chem. 1999, 71, 1997-2002.

12. Leavell, M. D.; Kruppa, G. H.; Leary, J. A. Determination of Phosphate Position in Hexose Monosaccharides Using an FTICR Mass sPectrometer: Ion/Molecule Reactions, Labeling Studies, and Dissociation Mechanisms. Int. J. Mass Spectrom. 2003, 222, 135-153.

13. Hashii, N.; Kawasaki, N.; Itoh, S.; Harazono, A.; Matsuishi, Y.; Hayakawa, T.; Kawanishi, T. Specific Detection of Lewis x-Carbohydrates in Biological Samples Using Liquid Chromatography/Multiple-stage Tandem Mass Spectrometry. Rapid Commun. Mass Spectrom. 2005, 19, 3315-3321.

14. Li, R.; Wu, Z. J.; Zhang, F.; Ding, L. S. Differentiation of Three Pairs of Aconite Alkaloid Isomers from Aconitum nagarum var. lasiandrum by Electrospray Ionization Tandem Mass Spectrometry. Rapid Commun. Mass Spectrom. 2006, 20, 157-170.

15. Bandu, M. L.; Desaire, H. The STEP Method (Statistical Test of Equivalent Pathways): Application to Pharmaceuticals. Analyst 2006, 131, 268-274.

16. Bandu, M. L.; Wilson, J.; Vachet, R. W.; Dalpathado, D. S.; Desaire, H. STEP (Statistical Test of Equivalent Pathways) Analysis: A Mass Spectrometric Method for Carbohydrates and Peptides. Anal. Chem. 2005, $77,5886-5893$.

17. Satterfield, M.; Brodbelt, J. S. Enhanced Detection of Flavonoids by Metal Complexation and Electrospray Ionization Mass Spectrometry. Anal. Chem. 2000, 72, 5898-5906.

18. Satterfield, M.; Brodbelt, J. S. Structural Characterization of Flavonoid Glycosides by Collisionally Activated Dissociation of Metal Complexes. J. Am. Soc. Mass Spectrom. 2001, 12, 537-549.

19. Zhang, J. M.; Brodbelt, J. S. Silver Complexation and Tandem Mass Spectrometry for Differentiation of Isomeric Flavonoid Diglycosides. Anal. Chem. 2005, 77, 1761-1770. 
20. Zhang, J. M.; Wang, J. M.; Brodbelt, J. S. Characterization of Flavonoids by Aluminum Complexation and Collisionally Activated Dissociation. J. Mass Spectrom. 2005, 40, 350-363.

21. Pikulski, M.; Brodbelt, J. S. Differentiation of Flavonoid Glycoside Isomers by Using Metal Complexation and Electrospray Ionization Mass Spectrometry. J. Am. Soc. Mass Spectrom. 2003, 14, 1437-1453.

22. Davis, B. D.; Brodbelt, J. S. Determination of the Glycosylation Site of Flavonoid Monoglucosides by Metal Complexation and Tandem Mass Spectrometry. J. Am. Soc. Mass Spectrom. 2004, 15, 1287-1299.

23. Davis, B. D.; Brodbelt, J. S. LC-MSn Methods for Saccharide Characterization of Monoglycosyl Flavonoids Using Postcolumn Manganese Complexation. Anal. Chem. 2005, 77, 1883-1890.

24. vanAcker, S. A. B. E.; vandenBerg, D. J.; Tromp, M. N. J. L.; Griffioen, D. H.; VanBennekom, W. P.; VanderVijgh, W. J. F.; Bast, A. Structural Aspects of Antioxidant Activity of Flavonoids. Free. Radic. Biol. Med. 1996, 20, 331-342.

25. Pietta, P. Flavonoids in Medicinal Plants; Marcel Dekker: New York, 1998

26. Paganga, G.; Miller, N.; Rice-Evans, C. A. The Polyphenolic Content of Fruit and Vegetables and Their Antioxidant Activities. What Does a Serving Constitute? Free. Radic. Res. 1999, 30, 153-162.

27. Jovanovic, S. V.; Steenken, S.; Tosic, M.; Marjanovic, B.; Simic, M. G. Flavonoids as Antioxidants. J. Am. Chem. Soc. 1994, 116, 4846-4851.

28. RiceEvans, C. A.; Miller, N. J. Antioxidant Activities of Flavonoids as Bioactive Components of Food. Biochem. Soc. Trans. 1996, 24, 790-795.

29. Hertog, M. G. L. Flavonoid Intake and Long-Term Risk of Coronary Heart-Disease and Cancer in the 7 Countries Study. Arch. Intern. Med. $1995,155,11841184$

30. Knekt, P.; Jarvinen, R.; Reunanen, A.; Maatela, J. Flavonoid Intake and Coronary Mortality in Finland: A Cohort Study. Br. Med. J. 1996, 312, 478-481.

31. Hollman, P. C. H.; Feskens, E. J. M.; Katan, M. B. Tea Flavonols in Cardiovascular Disease and Cancer Epidemiology. Proc. Soc. Exp. Biol. Med. 1999, 220, 198-202.

32. Mojzisova, G.; Kuchta, M. Dietary Flavonoids and Risk of Coronary Heart Disease. Physiol. Res. 2001, 50, 529-535.

33. Amic, D.; Davidovic-Amic, D.; Beslo, D.; Trinajstic, N. Structure-Radical Scavenging Activity Relationships of Flavonoids. Croat. Chem. Acta 2003, 76, 55-61.

34. Cao, G. H.; Sofic, E.; Prior, R. L. Antioxidant and Prooxidant Behavior of Flavonoids: Structure-Activity Relationships. Free. Radic. Biol. Med. 1997, 22, 749-760.

35. Bors, W. H. M. C. The Chemistry of Flavonoids. In Flavonoids in Health and Disease; C. A. Rice-Evans, Ed.; Marcel Dekker: New York, 1998; pp 111-136.
36. RiceEvans, C. A.; Miller, N. J.; Paganga, G. Structure-Antioxidant Activity Relationships of Flavonoids and Phenolic Acids. Free Radic. Biol. Med. 1996, 21, 417417.

37. Stobiecki, M. Application of Mass Spectrometry for Identification and Structural Studies of Flavonoid Glycosides. Phytochemistry 2000, 54, 237-256.

38. Cuyckens, H.; Claeys, M. Mass Spectrometry in the Structural Analysis of Flavonoids. J. Mass Spectrom. 2004, 39, 461461.

39. Nord, G. Some Properties of 2,2'-bipyridine, 1,10-Phenanthroline and Their Metal Complexes. Comments. Inorg. Chem. 1985, 4, 193-212.

40. Constable, E. C. The Reactions of Nucleophiles with Complexes of Chelating Heterocyclic Imines-A Critical Survey. Polyhedron 1983, 2, 551-572.

41. Brandt, W. W.; Dwyer, F. P.; Gyarfas, E. C. Chelate complexes of 1,10-phenanthroline and related compounds. Chem. Rev. 1954, 54, 959-1017.

42. McWhinnie, W. R.; Miller, J. D. Chemistry of Complexes Containing 2,2'-Bipyridyl, 1,10-Phenanthroline or 2,2',6',2'-Terpyridyl as Ligands. Adv. Inorg. Radiochem. 1969, 12, 135-173.

43. Martell, A. E.; Hancock, R. D. Metal Complexes in Aqueous Solutions, 1st ed.; Plenum Press: New York, 1996.

44. Satterfield, M.; Brodbelt, J. S. Relative Binding Energies of Gas-phase Pyridyl Ligand/Metal Complexes by Energy-Variable Collisionally Activated Dissociation in a Quadrupole Ion Trap. Inorg. Chem. 2001, 40, 5393-5400.

45. Wu, H. F.; Brodbelt, J. S. Gas-phase Chelation Reactions of Monopositive Cations with Heteroaromatic Ligands. Inorg. Chem. 1995, 34, 615-621.

46. Maerker, G. C. Synthesis of Some 4,4'-Disubstituted 2,2'-Bipyridines. J. Am. Chem. Soc. 1958, 80, 2745-2748.

47. Carlson, B.; Phelan, G. D.; Kim, J. H.; Jen, A. K. Y.; Dalton, L. R. Organic Light Emitting Devices Based upon Divalent Osmium Complexes. Abstr. Pap. Am. Chem. Soc. 2004, 227, U1314-U1314.

48. Ward, R. S.; Branciard, D.; Dignan, R. A.; Pritchard, M. C. Synthesis of C-2 Symmetric 2,2'-Bipyridyl Imidazolidinone and Oxazaborolidine Derivatives. Heterocycles 2002, 56, 157-170.

49. Denes, V.; Chira, R. Poly-Halogenation of Nitrogen Containing Heterocyclic-Compounds. 3. Polybromo-1,10-Phenanthrolines. J. Fur. Prak. Chem. 1978, 320, 172-175.

50. Zhang, J. M.; Brodbelt, J. S. Threshold Dissociation and Molecular Modeling of Transition Metal Complexes of Flavonoids. J. Am. Soc. Mass Spectrom. 2005, 16, 139-151. 